\title{
Synthesis and characterization of neomycin functionalized chitosan stabilized silver nanoparticles and study its antimicrobial activity
}

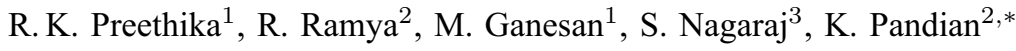 \\ ${ }^{1}$ Department of Chemistry, Thiagarajar College, Madurai-625009, India \\ ${ }^{2}$ Department of Inorganic Chemistry, University of Madras, Guindy Campus, Chennai-600025, India \\ ${ }^{3}$ CASBotany, University of Madras, Guindy Campus, Chennai-600025, India \\ *jeevapandian@yahoo.co.uk
}

PACS 82.65. $+\mathbf{r}$

DOI 10.17586/2220-8054-2016-7-4-759-764

\begin{abstract}
A simple green method was developed for the synthesis of silver nanoparticles in the presence of a neomycin-functionalized chitosan as stabilizing agent using a fresh lemon juice as green reducing agent. The stabilizing agent was synthesized based on the Schiff base formation reaction between the chitosan dialdehyde and neomycin antibiotic in $0.05 \mathrm{mM}$ at $\mathrm{pH} 7.0$. The combined form of neomycin antibiotic with chitosan can be used as stabilizing agent for silver nanoparticles (AgNPs) synthesized by a biogenic method using lemon juice as a green reducing agent. The neomycin functionalized chitosan stabilized AgNPs were characterized by various analytical techniques, including UVVisible spectra studies, FTIR, XRD and SEM. The antimicrobial activity of these composite was tested against human pathogenic Gram-positive and Gram-negative bacteria. The synergetic effect of the neomycin functionalized chitosan protected silver nanoparticles was tested against various drug resistant microorganisms. These chitosan derivatives can be used in combination with an anti-bacterial agent to treat and inhibit a resistant bacterial infection or the growth of resistant bacterial infection.
\end{abstract}

Keywords: silver nanoparticles, lemom juice, neomycin, chitosan, antimicrobial activity.

Received: 3 April 2016

Revised: 5 July 2016

\section{Introduction}

Due to its biocompatibility, biodegradability and non-toxicity, chitosan has increasingly been used in the biomedical and pharmaceutical fields [1-4]. Chemical modifications have been used to prepare chitosan derivatives with enhanced biological and physicochemical properties. Recently, aldehyde-functionalized chitosans have received considerable interest. The preparation of chitosan derivatives containing aldehyde groups have been achieved by reaction with periodates. The latter selectively cleaves the $2,3 \mathrm{C}-\mathrm{C}$ bond and converts chitosan into a dialdehyde derivative $[5,6]$. Aldehyde-functionalized chitosans have also been prepared by reaction with nitrous acid $\left(\mathrm{HNO}_{2}\right)$ [7-10]. In this method, chitosan undergoes deaminative cleavage of the 1, 4-glycosidic bonds, producing 2, 5-anhydro-D-mannose as the reducing end, which contain an aldehyde group. Hydrogen peroxide was also used to synthesis chitosan containing 2, 5-anhydro-D-mannose recently [11]. The periodate oxidation of chitosan was performed which yielded a dialdehyde chitosan (DAC) that can be used for the construction of biosensor and drug delivery applications [12]. Similarly, chitin and chitosan can be easily processed into hydrogels for various types of biomedical applications such as drug and gene delivery, wound healing and tissue engineering [13-15]. Drug loaded chitosan hydrogels have been generated using diffusion, entrapment and tethering techniques [16]. Chitosan membrane and its derivative have shown a prolonged antibacterial activity and decreased potential toxicity of silver $[17,18]$.

Recently, a facile and green method was reported in the literature to synthesize stable Ag nanoparticles (AgNPs) with a narrow size distribution. It is well established that chitosan (DAC) and its derivatives were widely used as both a reducing as well as stabilizing agent for the synthesis of metal nanoparticles. In the present study, we investigated the antibacterial activity of both neomycin-functionalized chitosan as well as the combined form of Neo-chit stabilized silver nanoparticles because of the synergetic microbial activity. Here, lemon juice was used to prepare narrow size silver nanoparticles, as previously reported in the literature.

\section{Materials and methods}

\subsection{Materials}

Analytical grades chitosan and sodium periodate were purchased from Sigma-Aldrich. The other reagents and solvents were analytical grade. All materials were used without further purification. 


\subsection{Preparation procedures}

2.2.1. Oxidation of chitosan. About $1 \mathrm{~g}$ chitosan $(5.34 \mathrm{mM})$ was dispersed in $50 \mathrm{ml} \mathrm{HCl}\left(10^{-3} \mathrm{M}\right)(\mathrm{pH}$ ranging from 4-5) with magnetic stirring. Then, $1 \mathrm{ml}$ aqueous sodium periodate $0.534 \mathrm{mM}$ was added, the reaction mixture was allowed to stir at $4{ }^{\circ} \mathrm{C}$ in the dark for 30 minutes. After completion of the reaction, to eliminate the unreacted periodate, $1 \mathrm{ml}$ of ethylene glycol was added. The oxidized chitosan was washed by distilled water and the sample was frozen and lyophilized for $4 \mathrm{~h}$.

2.2.2. Synthesis of neomycin functionalized chitosan (Neo-Chit). $10 \mathrm{ml}$ of chitosan dialdehyde mixed with $0.05 \mathrm{mM}$ of neomycin sulfate and then the reaction mixture was stirred for $2 \mathrm{~h}$. The powder form of the sample was isolated by by lyophilization after freezing with liquid nitrogen.

2.2.3. Synthesis of silver nanoparticles. $1 \mathrm{ml}$ of freshly prepared lemon juice was diluted with $3 \mathrm{ml}$ of distilled water and then the $\mathrm{pH}$ of the solution was adjusted to 11 . Then, $2 \mathrm{ml} 1 \mathrm{M}$ aq. $\mathrm{AgNO}_{3}$ was added dropwise to the above vigorously-stirred lemon juice solution. The polyphenol group present in the lemon juice reduced the silver ions to silver metal nanoparticles in th aq. medium. The appearance of reddish brown color solution indicated the formation of silver nanoparticles. Then, th silver nanoparticles were isolated via centrifugation.

2.2.4. Neomycin functionalized chitosan stabilized silver nanoparticles. To prepare Neo-Chit stabilized AgNPs, $0.1 \mathrm{~g}$ neomycin-functionalized chitosan was mixed with $1 \mathrm{ml}$ of silver nanoparticle solution and then allowed to stir for 30 min. The Neo-Chit @ AgNPs was isolated by centrifugation followed by drying under nitrogen atmosphere in the dark. The powdered samples were collected and stored in dark until further use Fig. 1.

2.2.5. Instrumentation. UV-Visible spectral studies were carried out by using a Shimadzu UV-Visible Spectrophotometer, Japan (Model UV-1800). The characterization of functional groups on the surface of AgNPs performed by FT-IR (perkin-Elmer, Germony) the spectra were scanned over 500 to $4000 \mathrm{~cm}^{-1}$.

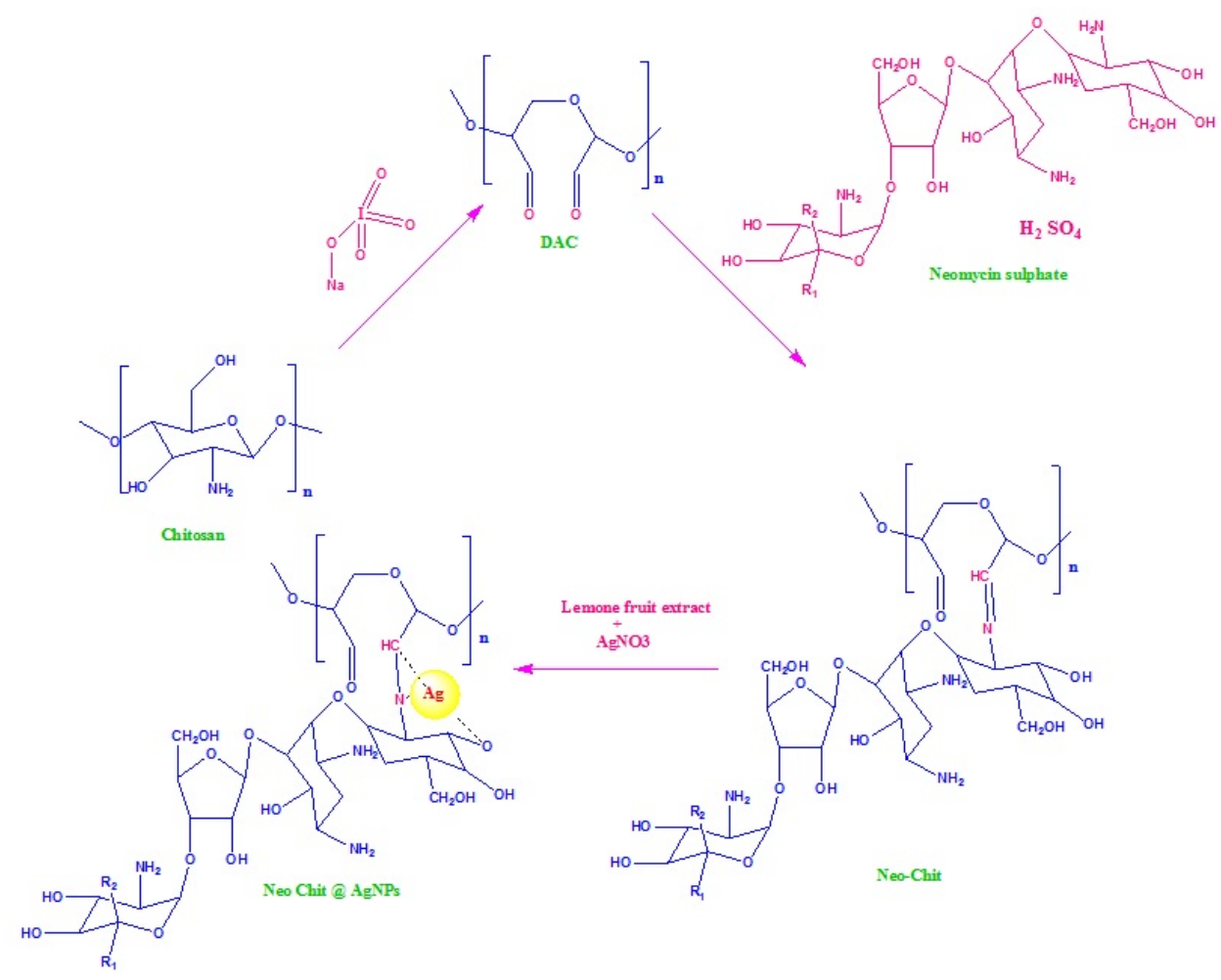

FIG. 1. Schematic representation of the synthetic route of Neomycin functionalized chitosan stabilized silver nanoparticles 


\section{Result and discussion}

\subsection{UV-Vis spectroscopy}

The UV-Visible spectrum of silver nanoparticles and Neo-chit protected AgNP are shown in Fig. 2. The Plasmon absorption peak was observed at $408 \mathrm{~nm}$ which indicated the formation of silver nanoparticles. From the UV-Visible spectral studies, it is clearly seen that the particle size of the silver nanoparticles ranged from 5-20 nm. The peak shape of AgNPs is narrow and sharp, providing evidence for the uniform size distribution of the silver nanoparticles in lemon juice solution. The appearance a sharp Plasmon band is due to the formation of silver nanoparticles having a narrow size range. A slight shift in Plasmon band position is due to the surface modification of silver nanoparticles using neomycin-functionalized chitosan as stabilizing agent. The decrease in Plasmon band position is due to the surface modification of the neomycin functionalized AgNP. The broaderning of the peak position was also observed which is due to the partial aggregated particles of the silver nanoparticles.

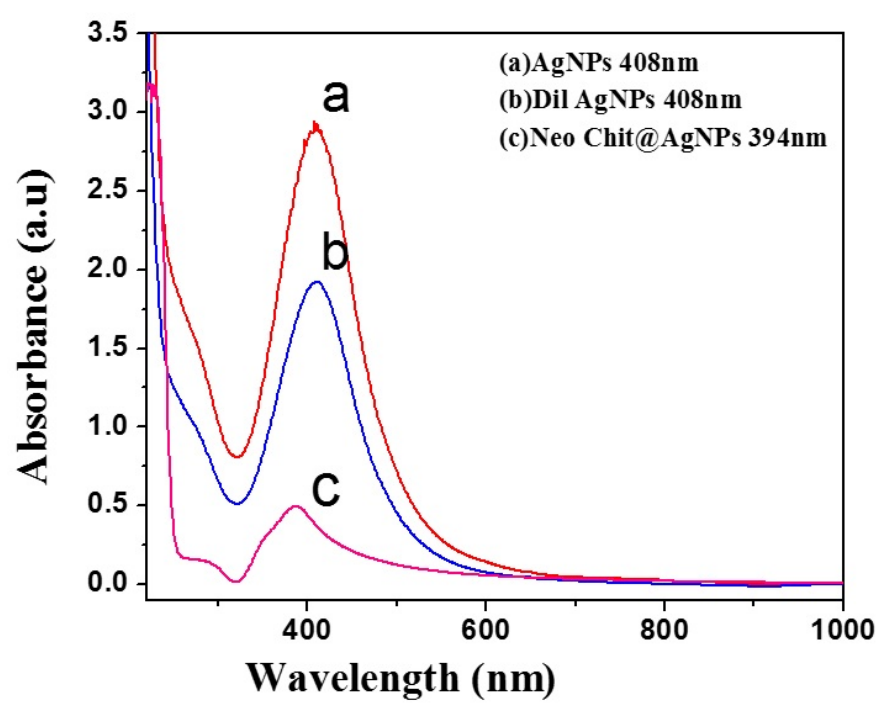

FIG. 2. (a) UV-Visible spectrum of AgNPs obtained from lemon juice reduction method; (b Dilute AgNPs and (c) Neo-chit @ AgNPs

\subsection{FT-IR-spectrum of neomycin functionalized chitosan modified AgNPs}

The FT-IR spectrum was recorded for both neomycin-functionalized chitosan and neomycin-functionalized chitosan protected silver nanoparticles. The major bands at 2926, 2851, 1746, 881, $774 \mathrm{~cm}^{-1}$ are assigned for the characteristic bands for aldehyde group that resulted from periodate mediated oxidation was observed at Fig. 3(b). The bands at 1077 and $1020 \mathrm{~cm}^{-1}$ are typical for neomycin-functionalized chitosan modified AgNPs was observed at Fig. 3(c), Table 1.

TABLE 1. FT-IR bands related to chitosan/modified chitosans and their respective assignments

\begin{tabular}{|c|c|c|c|}
\hline FT-IR band $\left(\mathbf{c m}^{-1}\right)$ & Assignment & FT-IR band(cm & \\
& -1 & ) & Assignment \\
\hline 3356,2926 & N-H,C-H & 1071,1020 & C-O-C \\
\hline 3425 & $\mathrm{O}-\mathrm{H}$ & 2352,1323 & N-N,NO ${ }_{2}$ \\
\hline 2851 & $\mathrm{C}-\mathrm{H}$ & 2075 & $\mathrm{O}-\mathrm{H}$ \\
\hline 1746 & $\mathrm{C}=\mathrm{O}$ & 1077 & $\mathrm{C}-\mathrm{O}$ \\
\hline 1626 & $\mathrm{C}-\mathrm{C}$ & 881,774 & $\mathrm{C}-\mathrm{H}, \mathrm{NO}_{3}$ \\
\hline 1406 & $\mathrm{C}=\mathrm{O}$ & & \\
\hline
\end{tabular}




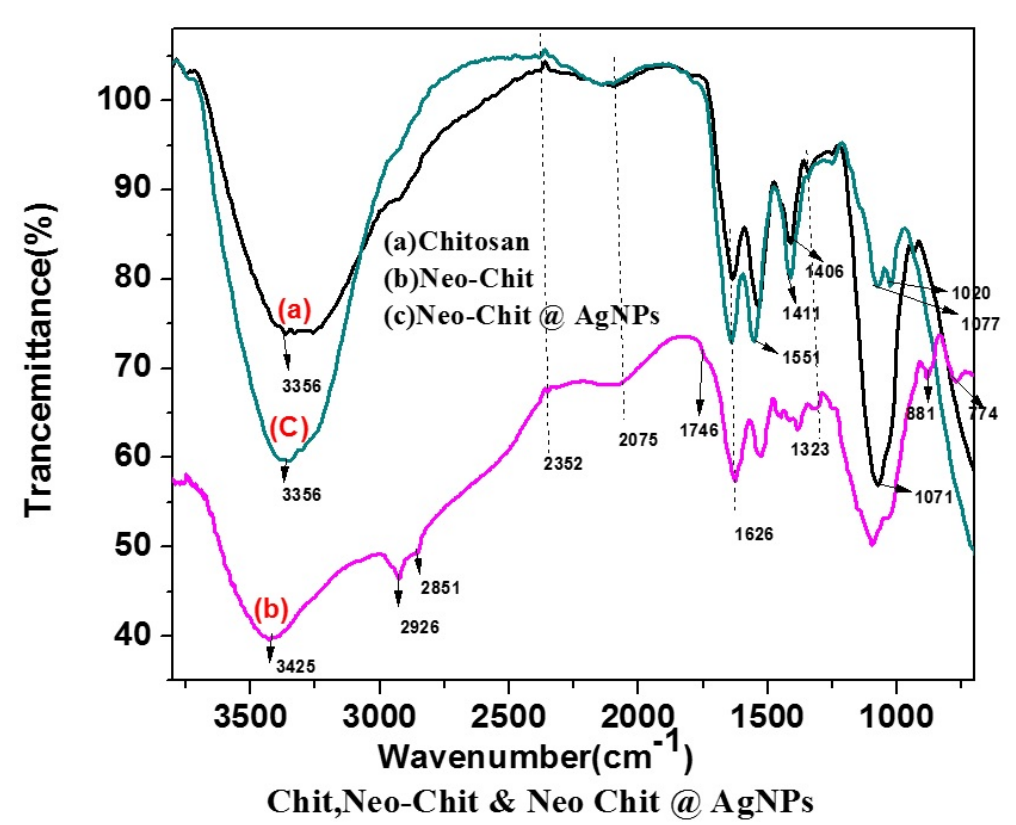

FIG. 3. FT-IR spectrum of chitosan and neomycin functionalized chitosan

\subsection{Scanning Electron Microscopic studies}

Conventional high vacuum scanning electron microscopy (SEM) images were also taken to envision the structure of oxidized chitosan and Chitosan dialdehyde with neomycine@AgNPs. Fig. 4 shows that there is no change for the stretched out and fibrous network of chitosan, but on the surface of oxidized chitosan one can see a slight degradation of some foliage. The EDX pattern of chitosan dialdehyde with neomycin @ AgNPs is shown in Fig. 5. This pattern shows peaks for the elements $\mathbf{A g}$ and $\mathbf{C}$ are the major components. From these studies, we conclude that the the chitosan dialdehyde-functionalized neomycin stabilizes the AgNPs.
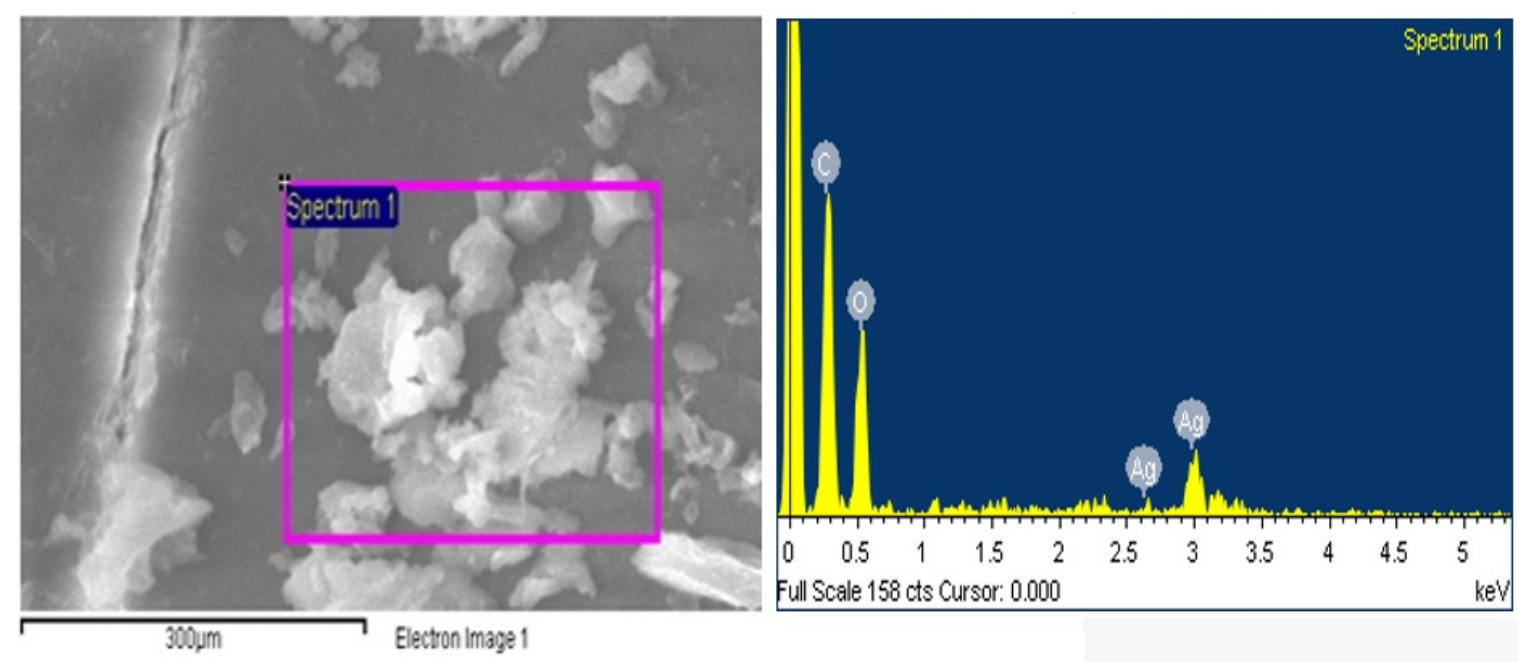

FIG. 4. SEM - EDAX of Chitosan dialdehyde with neomycin@ AgNPs

\subsection{Antibacterial activity of Neo-Chit @ AgNP}

It is well established that silver nanoparticles with various capping agents have shown excellent antimicrobial activity $[18,19]$. In the present study, we demonstrate the antimicrobial activity of silver nanoparticles, neomycinfunctionalized chitosan modified AgNPs against various Gram negative and Gram positive bacteria. The disc diffusion method was used for the quantification of inactivation of microbial growth. From the experimental 


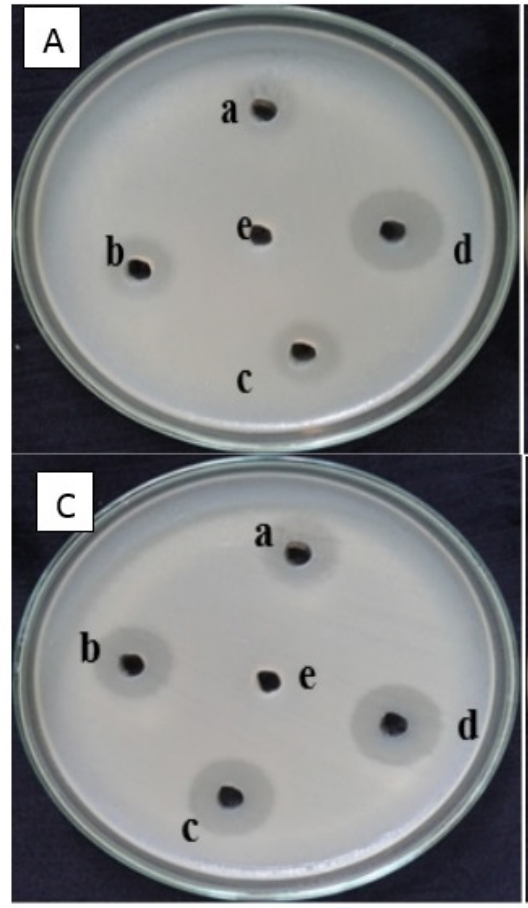

$\mathrm{a}-25 \mu \mathrm{g} / \mathrm{ml}$ of $\mathrm{Neo}-\mathrm{Chi}$ $\mathrm{b}-50 \mu \mathrm{g} / \mathrm{ml}$ of Neo-Chi $\mathrm{c}-75 \mu \mathrm{g} / \mathrm{ml}$ of Neo-Chi $\mathrm{d}-25 \mu \mathrm{g} / \mathrm{ml}$ (Standard) chloromphenicol $\mathrm{e}-50 \mu \mathrm{g} / \mathrm{ml} 0.1 \%$ DMSO
B

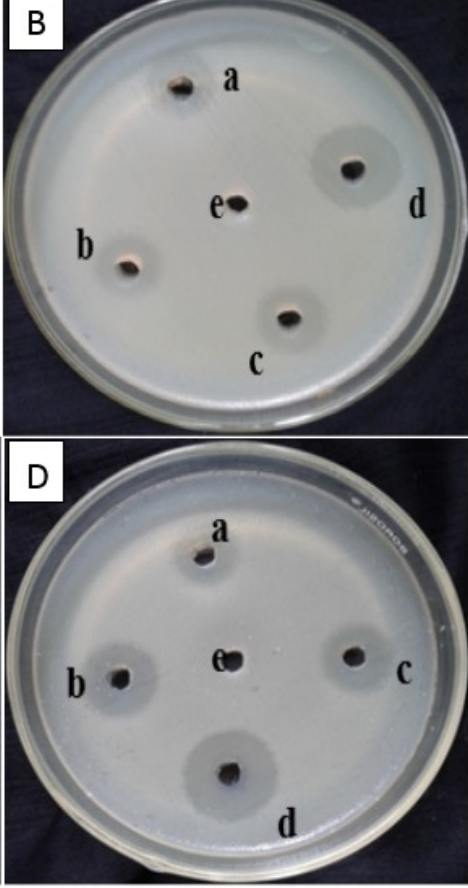

a $-25 \mu \mathrm{g} / \mathrm{ml}$ Neo-Chit@, AgNPs

b-50 $\mu \mathrm{g} / \mathrm{ml}$ Neo-Chit@AgNPs

c-75 $\mu \mathrm{g} / \mathrm{ml}$ Neo-Chit@AgNPs

$\mathrm{d}-25 \mu \mathrm{g} / \mathrm{ml}$ (Standard) chloromphenicol

$\mathrm{e}-50 \mu \mathrm{g} / \mathrm{ml} 0.1 \%$ DMSO

FIG. 5. Antibacterial activity of the Neo-chit and Neo-chit@AgNPs against A, C - Staphylococcus aureus and B, D - Salmonella typhi

studies it is inferred that Neo-chit@AgNPs have shown an enhanced antimicrobial activity than the Neo-chit as well as bare AgNP (Table 2).

TABle 2. Antimicrobial activity of the Neo-chit and Neo-chit @ AgNPs against Gram positive and Gram negative bacteria

\begin{tabular}{|c|c|c|c|c|c|c|c|c|c|c|}
\hline & \multicolumn{4}{|c|}{ Gram positive bacteria } & \multicolumn{6}{c|}{ Gram negative bacteria } \\
\hline \multirow{2}{*}{ Sample $(\mu \mathbf{g} / \mathbf{m l})$} & \multicolumn{3}{|c|}{ Staphylococcus aureus } & \multicolumn{5}{|c|}{ Salmonella typhi } \\
\cline { 2 - 11 } & a & b & c & d & e & a & b & c & d & e \\
\hline Neo-chit & 2.5 & 3 & 3.5 & 8 & - & - & - & - & 11 & - \\
\hline Neo-chit@AgNPs & 3 & 6 & 8 & 14 & - & 4 & 6 & 9 & 24 & - \\
\hline
\end{tabular}

\section{Conclusion}

We have synthesized neomycin antibiotic drug-functionalized chitosan by a Schiff base reaction using chitosan dialdehyde. The neomycin-functionalized chitosan was isolated in pure form by dialysis followed by freeze drying method. From the FT-IR result it is confirmed that neomycin aminoglucoside antibiotic drug molecules effective functionalized on chitosan backbone. The neomycin-functionalized chitosan was used further to stabilize the green synthesized silver nanoparticles using lemon juice as reducing agent. The antibacterial activity of the Neo-chit and Neo-chit@AgNP were tested. An enhanced antimicrobial activity was noted in the case of neo-chit-stabilized silver nanoparticles system than the neo-chit system and silver nanoparticles. The combined form of silver nanoparticles and Neo-Chit showed can be used to eradicate the microbial drug resistance organisms. 


\section{References}

[1] Enxian Lu, Scott Franzblan, Carmen Popesu. Preparation of aminoglycoside-loaded chitosan nanoparticles using dextran sulphate as a counter ion. J. Micro encapsulation: Micro and Nano carriers, 2009, 26(4), P. 346-354.

[2] Jayakumar R., Deepthy Menon, Manzoor K., Tamura H. Biomedical application of chitin and chitosan based nanomaterials. Carbohydrate polymers, 2010, 82(2), P. 227-232.

[3] Chen J., Liu Q., Zhang X., Zhang Q. Prevaporation and characterization of chitosan membranes cross linked by 3aminopropyltriethoxysilane. J. Member. Sci, 2007, 292, P. 125-132.

[4] Ravi Kumar M.N.V. A review of chitin and chitosan applications. Reactive and Functional Polymers, 2000,46, P. 1-27.

[5] Vold I.M., Christensen B.E. Periodate oxidation of chitosans with different chemicalCompositions. Carbohydr Res, 2005, 340, P. 679-684.

[6] Xin Liu, Yunhui. Preparation process and Antimicrobial Properties of cross-linking chitosan on to periodate-oxidised bamboo pulp fabric. J. of Fibers and polymers, 2014, 15, P. 1887-1894.

[7] Jolanta kumirska, Mirko X., Weinhold, Jorg Thoming, Piotr Stepnowski. Biomedical Activity of Chitin/Chitosan Based Materials- Influence of properties Apart from Molecular Weight and Degree of N-Acetylation. J. Polymer, 2011, 3, P. 1875-1901.

[8] Hilde K. Holme, Lene Davidsen, Are Kristiansena. Kinetics and mechanism of depolymerization of alginate and chitosan in aqueous solution. Carbohydrate polymers, 2007, 73, P. 656-664.

[9] Tommeraas K., Varum K.M., Christensen B.E., Smidsrod O. Preparation and characterisation of oligosaccharides produced by nitrous acid depolymerisation of chitosans. Carbohydr. Res, 2001, 333, P. 137-144.

[10] Lin C.W., Lin J.C. Characterization and blood coagulation evaluation of the water soluble chitooligosaccharides prepared by a facile fractionation method. Biomacromolecules, 2003, 4, P. 1691-1697.

[11] Tian F., Liu Y., Hu K., Zhao B. Study of the depolymerization behavior of chitosan by hydrogen peroxide. Carbohydr. Polym., 2004, 57, P. 31-37.

[12] Yan Feng, Limin Yang, Feng Li. A novel sensing platform based on periodate-oxidized chitosan. Anal. Methods, (2010), 2, P. 2011-2016.

[13] Jayakumar R., Divya Rani V.V., Shalumon K.T., Sudheesh Kumar P.T. Nair S.V., Furuike T., et al. Bioactive and osteoblast cell attachement studies of novel $\alpha$-and $\beta$-chitinmembranes for tissue engineering applications. Int. J. Biol Macromol, 2009,45 , P. $260-4$.

[14] Jayakumar R., Prabaharan M., Reis R.L., Mano J.F. Graft copolymerized chitosan - present status and applications. Carbohydr. Polym., 2005, 62, P. 142-58.

[15] Prabaharan M., Mano J.F. Chitosan-based particles as controlled drug delivery systems. Drug Deliv, 2005,12, P. $41-57$.

[16] Lin C.C., Anseth K.S. PEG hydrogels for the controlled release of biomolecules in regenerative medicine. Pharm. Res, 2009 , 26, P. 631-43.

[17] Mi F.L., Wu Y.B., Shyu S.S., Chao A.C., Lai J.Y., Su C.C. Asymmetric chitosan membranes prepared by dry/wet phase separation: a new type of wound dressing for controlled antibacterial release. J. Membr Sci, 2003, 212, P. $237-54$.

[18] Sharma V.K., Yangard R.A. Green Synthesis and their antimicrobial activities. J. Colloid Interface. Sci, 2009 , 9, P. 83-96.

[19] Kora A.J., Rastogi L. Enhancement of antibacterial activity of capped silver nanoparticles in combination with antibiotics, on model Gram-negative and Gram-positive bacteria. Bioinorg. Chem. Appl, 2013, P. 1-7. 